\title{
Studies Towards the Synthesis of Oximidines I and II
}

(Supporting Information)

\author{
Torsten Haack, ${ }^{\S}$ Serdar Kurtkaya, ${ }^{\dagger}$ James P. Snyder, ${ }^{\dagger *}$ and Gunda I. Georg ${ }^{\S^{*}}$ \\ ${ }^{\S}$ Department of Medicinal Chemistry, University of Kansas, 1251 Wescoe Hall Drive, Lawrence, KS \\ 66045-7582, USA; ${ }^{\dagger}$ Department of Chemistry, Emory University, 1515 Pierce Drive, \\ Atlanta, GA 30322, USA
}

\section{General remarks :}

${ }^{1} \mathrm{H}$ NMR and ${ }^{13} \mathrm{C}$ spectra were determined on a $400 \mathrm{MHz}$ NMR. Mass spectra were obtained from a ZAB HS mass spectrometer equipped with an 11/250 data system. Fast atom bombardment mass spectrometry (FABMS) experiments were performed with a Xenon gun operated at $8 \mathrm{KeV}$ energy and $0.8 \mathrm{~mA}$ emission. Fast atom bombardment high resolution mass spectra (FAB HRMS) were recorded at $1: 10,000$ resolution using linear voltage scans under data system control and the data were collected in a multichannel analyzer mode. A FT-IR spectrometer was used to record infrared spectra. Optical rotations were obtained at room temperature. Melting points are uncorrected. THF was dried by distillation from sodium/benzophenone. Methylene chloride was dried by distillation from calcium hydride. All other dry solvents and chemicals were obtained commercially. Tetrakis(triphenylphosphine)-palladium(0) was purchased from STREM chemicals. All reactions involving dry solvents were carried out under an argon atmosphere. Extractive workups were carried out using the solvents mentioned in the procedures and water (3 extractions). The combined organic layers were dried over magnesium sulfate, filtered and the filtrate was concentrated under reduced pressure. Column chromatography was performed on silica gel (silica gel 60, 0.040-0.063 mm, 230-400 mesh ASTM) with the solvent systems indicated in the procedures.

\section{4,4-Diethoxybut-2-ynoic Acid Methyl Ester (9). ${ }^{1}$}

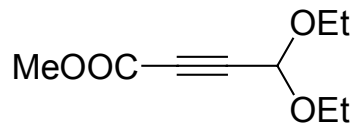

To an ice-cooled solution of propiolaldehyde diethylacetal $(11.2 \mathrm{~mL}, 10 \mathrm{~g}, 78 \mathrm{mmol})$ in dry THF $(30 \mathrm{~mL})$ was added dropwise methyllithium (1.4 $\mathrm{M}$ in $\left.\mathrm{Et}_{2} \mathrm{O}, 61 \mathrm{~mL}, 86 \mathrm{mmol}\right)$. When the addition was complete, the resulting mixture was added dropwise to a solution of methyl chloroformate $(6.7 \mathrm{~mL}, 8.2 \mathrm{~g}, 87 \mathrm{mmol})$ in dry THF $(30 \mathrm{~mL})$ at $-78{ }^{\circ} \mathrm{C}$. The resulting mixture was allowed to reach room temperature and was then stirred at this temperature overnight. Extractive workup with ether followed by distillation $\left(52{ }^{\circ} \mathrm{C}, 0.1\right.$ Torr) afforded $9.9 \mathrm{~g}(68 \%)$ of ester 9 as a colorless oil. ${ }^{1} \mathrm{H} \mathrm{NMR}\left(400 \mathrm{MHz}, \mathrm{CDCl}_{3}\right): \delta$ $5.35(\mathrm{~s}, 1 \mathrm{H}), 3.78(\mathrm{~s}, 3 \mathrm{H}), 3.74\left(\mathrm{dq}, 2 \mathrm{H},{ }^{3} J=7.1 \mathrm{~Hz},{ }^{3} J=2.3 \mathrm{~Hz}\right), 3.61\left(\mathrm{dq}, 2 \mathrm{H},{ }^{3} J=7.0\right.$ 
$\left.\mathrm{Hz},{ }^{3} J=2.2 \mathrm{~Hz}\right), 1.23\left(\mathrm{t}, 6 \mathrm{H},{ }^{3} J=7.0 \mathrm{~Hz}\right) ;{ }^{13} \mathrm{C} \mathrm{NMR}\left(100 \mathrm{MHz}, \mathrm{CDCl}_{3}\right): \delta 153.3,90.8$, $81.4,75.8,61.5,52.9,14.9$.

\section{2-Diethoxymethyl-6-methoxybenzoic Acid Methyl Ester (11). ${ }^{1}$}<smiles>CCOC(OCC)c1cccc(OC)c1C(=O)OC</smiles>

A mixture of alkyne $9(573 \mathrm{mg}, 3.08 \mathrm{mmol})$ and 1-methoxy-1,4-cyclohexadiene $(6.0 \mathrm{~g}$, $54.4 \mathrm{mmol}$ ) was heated at $170{ }^{\circ} \mathrm{C}$ under reflux overnight. The resulting mixture was directly subjected to column chromatography (EtOAc/hexanes $2: 8)$ to give $665 \mathrm{mg}(80 \%)$ of compound 11 as a colorless oil. ${ }^{1} \mathrm{H}$ NMR $\left(400 \mathrm{MHz}, \mathrm{CDCl}_{3}\right): \delta 7.35\left(\mathrm{t}, 1 \mathrm{H},{ }^{3} \mathrm{~J}=8.0\right.$ $\mathrm{Hz}), 7.22\left(\mathrm{~d}, 1 \mathrm{H},{ }^{3} J=7.8 \mathrm{~Hz}\right), 6.90\left(\mathrm{~d}, 1 \mathrm{H},{ }^{3} \mathrm{~J}=7.1 \mathrm{~Hz}\right), 5.65(\mathrm{~s}, 1 \mathrm{H}), 3.90(\mathrm{~s}, 3 \mathrm{H}), 3.83$ $(\mathrm{s}, 3 \mathrm{H}), 3.49-3.59(\mathrm{~m}, 4 \mathrm{H}), 1.21\left(\mathrm{t}, 6 \mathrm{H},{ }^{3} J=7.1 \mathrm{~Hz}\right) ;{ }^{13} \mathrm{C} \mathrm{NMR}\left(100 \mathrm{MHz}, \mathrm{CDCl}_{3}\right): \delta$ $168.3,156.4,138.0,130.2,122.2,118.8,111.1,99.0,61.2,56.0,52.2,15.0$.

\section{3-Hydroxy-7-methoxy-3H-isobenzofuran-1-one (12). ${ }^{1}$}

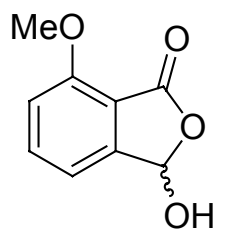

Compound 11 (463 mg, $1.73 \mathrm{mmol}$ ) was heated in a mixture of AcOH (2 mL) and $2 \mathrm{~N} \mathrm{HCl}(2 \mathrm{~mL})$ at reflux temperature overnight. Extractive workup with ethyl acetate and recrystallization from water furnished $188 \mathrm{mg}(61 \%)$ of 12 as colorless needles.

2-(2-Iodovinyl)-6-methoxybenzoic Acid 4-Methoxybenzyl Ester (13).<smiles>COc1ccc(COC(=O)c2c(/C=C/I)cccc2OC)cc1</smiles>

A mixture of 12 (970 mg, $5.38 \mathrm{mmol}), p$-methoxybenzyl chloride $(1.1 \mathrm{~mL}, 1.27 \mathrm{~g}$, $8.1 \mathrm{mmol})$ and diisopropylethylamine $(1.4 \mathrm{~mL}, 1.04 \mathrm{~g}, 8.1 \mathrm{mmol})$ in dry DMF $(10 \mathrm{~mL})$ was heated at $100{ }^{\circ} \mathrm{C}$ overnight. Extractive workup of the resulting mixture with ether gave the crude aldehyde which was dissolved together with iodoform (4.24 g, $10.8 \mathrm{mmol})$ in dry THF $(10 \mathrm{~mL})$. This solution was added dropwise to an ice-cooled suspension of chromium(II)-chloride $(3.97 \mathrm{~g}, 32.3 \mathrm{mmol})$ in dry THF $(30 \mathrm{~mL})$. The resulting red mixture was stirred at $0{ }^{\circ} \mathrm{C}$ for $5 \mathrm{~h}$ and after this time it was quenched with water. Extractive workup with ether and column chromatography (EtOAc/hexanes 2:8, slower moving spot) yielded $1.24 \mathrm{~g}(54 \%)$ of compound $\mathbf{1 3}$ as a slightly yellow oil and $0.31 \mathrm{~g}$ 
(14\%) of its cis-isomer (faster moving spot) as a slightly yellow oil: IR (neat) 2957, 2836, $1727,1515,1471,1270,1247,1110,1065,827,762 \mathrm{~cm}^{-1} ;{ }^{1} \mathrm{H}$ NMR $\left(400 \mathrm{MHz}, \mathrm{CDCl}_{3}\right)$ : $\delta 7.40\left(\mathrm{~d}, 2 \mathrm{H},{ }^{3} J=8.5 \mathrm{~Hz}\right), 7.28\left(\mathrm{~d}, 1 \mathrm{H},{ }^{3} J=14.7 \mathrm{~Hz}\right), 7.28\left(\mathrm{t}, 1 \mathrm{H},{ }^{3} J=6.4 \mathrm{~Hz}\right), 6.95(\mathrm{~d}$, $\left.1 \mathrm{H},{ }^{3} J=7.9 \mathrm{~Hz}\right) 6.93\left(\mathrm{~d}, 2 \mathrm{H},{ }^{3} J=8.6 \mathrm{~Hz}\right), 6.84\left(\mathrm{~d}, 1 \mathrm{H},{ }^{3} J=8.3 \mathrm{~Hz}\right), 6.80\left(\mathrm{~d}, 1 \mathrm{H},{ }^{3} J=\right.$ $14.8 \mathrm{~Hz}), 5.32(\mathrm{~s}, 2 \mathrm{H}), 3.81(\mathrm{~s}, 3 \mathrm{H}), 3.80(\mathrm{~s}, 3 \mathrm{H}) ;{ }^{13} \mathrm{C} \mathrm{NMR}\left(100 \mathrm{MHz}, \mathrm{CDCl}_{3}\right): \delta 167.2$, 159.6, 156.4, 141.4, 136.2, 130.5, 130.4, 127.5, 121.6, 117.7, 114.0, 110.6, 80.2, 67.1, 55.9, 55.2; HRMS (FAB+) calcd for $\mathrm{C}_{18} \mathrm{H}_{17} \mathrm{O}_{4} \mathrm{I}+\mathrm{H} 425.0259$ found 425.0263 .

(1S,4S)-2,2-Dimethylpropionic Acid 1-(2,2-Dimethyl-[1,3]dioxolan-4-yl)-3,3-bisethylsulfanylpropyl Ester.

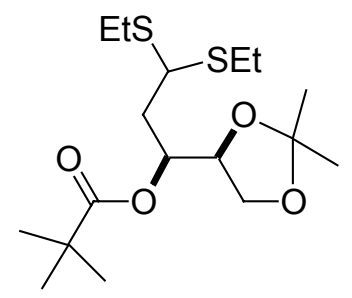

A mixture of $15^{2,3}$ (12.9 g, $\left.46 \mathrm{mmol}\right)$, pivaloyl chloride ( $18 \mathrm{~mL}, 17.6 \mathrm{~g}, 146 \mathrm{mmol}$ ), triethylamine $(20 \mathrm{~mL}, 14.5 \mathrm{~g}, 143 \mathrm{mmol})$ and DMAP $(500 \mathrm{mg}, 4 \mathrm{mmol})$ in dry methylene chloride $(100 \mathrm{~mL})$ was heated at reflux temperature for $16 \mathrm{~h}$. After cooling to room temperature the mixture was neutralized with sat. $\mathrm{K}_{2} \mathrm{CO}_{3}$. Extractive workup with ether and column chromatography (EtOAc/hexanes 2:8) afforded $15.1 \mathrm{~g} \mathrm{(90 \% )} \mathrm{of} \mathrm{the} \mathrm{pivaloate}$ as a colorless oil: $[\alpha]_{\mathrm{D}}-10.9$ (c 1.3, $\mathrm{CHCl}_{3}$ ); IR (neat) 2972, 2931, 2873, 1730, 1370, $1281,1156,1065,974,856 \mathrm{~cm}^{-1} ;{ }^{1} \mathrm{H}$ NMR $\left(400 \mathrm{MHz}, \mathrm{CDCl}_{3}\right): \delta 5.27$ (ddd, $1 \mathrm{H},{ }^{3} J=8.7$ $\left.\mathrm{Hz},{ }^{3} J=3.9 \mathrm{~Hz},{ }^{3} J=3.9 \mathrm{~Hz}\right), 4.20-4.24(\mathrm{~m}, 1 \mathrm{H}), 3.97\left(\mathrm{dd}, 1 \mathrm{H},{ }^{3} J=8.6 \mathrm{~Hz},{ }^{3} J=6.8 \mathrm{~Hz}\right)$, $3.78\left(\mathrm{dd}, 1 \mathrm{H},{ }^{3} J=9.4 \mathrm{~Hz},{ }^{3} J=5.5 \mathrm{~Hz}\right), 3.70\left(\mathrm{dd}, 1 \mathrm{H},{ }^{3} J=8.6 \mathrm{~Hz},{ }^{3} J=5.9 \mathrm{~Hz}\right), 2.52-$ $2.72(\mathrm{~m}, 4 \mathrm{H}), 2.20\left(\mathrm{ddd}, 1 \mathrm{H},{ }^{2} J=14.5 \mathrm{~Hz},{ }^{3} J=8.8 \mathrm{~Hz},{ }^{3} J=5.5 \mathrm{~Hz}\right), 2.04\left(\mathrm{ddd}, 1 \mathrm{H},{ }^{2} J=\right.$ $\left.14.5 \mathrm{~Hz},{ }^{3} J=9.4 \mathrm{~Hz},{ }^{3} \mathrm{~J}=4.0 \mathrm{~Hz}\right), 1.42(\mathrm{~s}, 3 \mathrm{H}), 1.31(\mathrm{~s}, 3 \mathrm{H}), 1.24\left(\mathrm{t}, 3 \mathrm{H},{ }^{3} J=7.4 \mathrm{~Hz}\right)$, $1.24\left(\mathrm{t}, 3 \mathrm{H},{ }^{3} J=7.4 \mathrm{~Hz}\right), 1.21(\mathrm{~s}, 9 \mathrm{H}) ;{ }^{13} \mathrm{C}$ NMR $\left(100 \mathrm{MHz}, \mathrm{CDCl}_{3}\right): \delta 177.9,109.4$, 75.9, 70.4, 65.2, 47.3, 38.9, 36.9, 27.1, 26.2, 25.1, 24.0, 23.9, 14.3, 14.3; HRMS (ES+) calcd for $\mathrm{C}_{17} \mathrm{H}_{32} \mathrm{O}_{4} \mathrm{~S}_{2}+\mathrm{NH}_{4} 382.2086$ found 382.2110 . 
(1S,2S)-2,2-Dimethylpropionic Acid 1-(2,2-Bis-ethylsulfanylethyl)-2,3dihydroxypropyl Ester (16).

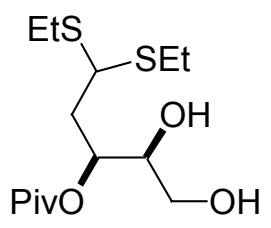

(1S,4S)-2,2-Dimethylpropionic acid 1-(2,2-dimethyl-[1,3]dioxolan-4-yl)-3,3-bisethylsulfanylpropyl ester $(155 \mathrm{mg}, 0.43 \mathrm{mmol})$ was dissolved in $70 \%$ acetic acid $(8 \mathrm{~mL})$ and the solution was kept at room temperature overnight. After neutralization with sat. $\mathrm{K}_{2} \mathrm{CO}_{3}$ the compound was extracted with ether. Column chromatography (EtOAc/hexanes 1:1) gave $122 \mathrm{mg}(88 \%)$ of the desired diol $\mathbf{1 6}$ as a colorless oil: $[\alpha]_{\mathrm{D}}-4.2\left(\mathrm{c} 1.4, \mathrm{CHCl}_{3}\right)$; IR (neat) $3428,2969,2929,2872,1731,1480,1456,1283$, $1156,1036,757 \mathrm{~cm}^{-1} ;{ }^{1} \mathrm{H}$ NMR $\left(400 \mathrm{MHz}, \mathrm{CDCl}_{3}\right): \delta 5.26\left(\mathrm{ddd}, 1 \mathrm{H},{ }^{3} J=9.0 \mathrm{~Hz},{ }^{3} J=\right.$ $\left.3.8 \mathrm{~Hz},{ }^{3} J=3.8 \mathrm{~Hz}\right), 3.70-3.80(\mathrm{~m}, 2 \mathrm{H}), 3.44-3.55(\mathrm{~m}, 2 \mathrm{H}), 2.70-2.80(\mathrm{~m}, 2 \mathrm{H}), 2.53-2.70$ (m, 4H), 2.26 (ddd, $1 \mathrm{H},{ }^{2} J=14.4 \mathrm{~Hz},{ }^{3} J=9.2 \mathrm{~Hz},{ }^{3} J=5.2 \mathrm{~Hz}$ ), 2.06 (ddd, $1 \mathrm{H},{ }^{2} J=14.2$ $\left.\mathrm{Hz},{ }^{3} J=9.7 \mathrm{~Hz},{ }^{3} J=4.1 \mathrm{~Hz}\right), 1.23\left(\mathrm{t}, 3 \mathrm{H},{ }^{3} J=7.4 \mathrm{~Hz}\right), 1.22\left(\mathrm{t}, 3 \mathrm{H},{ }^{3} J=7.4 \mathrm{~Hz}\right), 1.21(\mathrm{~s}$, 9H). ${ }^{13} \mathrm{C}$ NMR (100 MHz, $\left.\mathrm{CDCl}_{3}\right): \delta 178.9,72.9,71.8,63.2,47.2,39.0,36.8,27.1,24.1$, 23.7, 14.4, 14.3; HRMS (FAB+) calcd for $\mathrm{C}_{14} \mathrm{H}_{28} \mathrm{O}_{4} \mathrm{~S}_{2}\left(\mathrm{M}^{+}\right) 324.1429$ found 324.1426 .

\section{(1S,2S)-2,2-Dimethylpropionic Acid 1-(2,2-Bis-ethylsulfanylethyl)-3-tert- butyldimethylsilanyloxy)-2-hydroxypropyl Ester.}

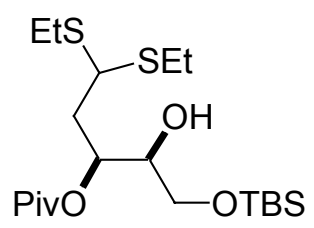

To a solution of $\mathbf{1 6}$ (4.69 g, $14.4 \mathrm{mmol})$ and imidazole (1.46 g, $21.4 \mathrm{mmol})$ in dry DMF $(5 \mathrm{~mL})$ was added tert-butyldimethylsilyl chloride $(2.45 \mathrm{~g}, 16.3 \mathrm{mmol})$ and the mixture was stirred for $1 \mathrm{~h}$ after which time water was added. Extractive workup with ether followed by column chromatography (EtOAc/hexanes 1:9) afforded $5.36 \mathrm{~g}(85 \%)$ of the desired TBS-ether as a colorless oil. IR (neat) 3500, 2929, 1731, 1463, 1282, 1257, 1156, $838,777,669 \mathrm{~cm}^{-1} ;{ }^{1} \mathrm{H}$ NMR $\left(400 \mathrm{MHz}, \mathrm{CDCl}_{3}\right): \delta 5.29\left(\mathrm{ddd}, 1 \mathrm{H},{ }^{3} J=8.5 \mathrm{~Hz},{ }^{3} J=4.4\right.$ $\left.\mathrm{Hz},{ }^{3} J=4.4 \mathrm{~Hz}\right), 3.80\left(\mathrm{dd}, 1 \mathrm{H},{ }^{3} J=8.9 \mathrm{~Hz},{ }^{3} J=5.9 \mathrm{~Hz}\right), 3.69-3.75(\mathrm{~m}, 1 \mathrm{H}), 3.64(\mathrm{dd}$, $\left.1 \mathrm{H},{ }^{3} J=10.2 \mathrm{~Hz},{ }^{3} J=4.0 \mathrm{~Hz}\right),\left(\mathrm{dd}, 1 \mathrm{H},{ }^{3} J=10.0 \mathrm{~Hz},{ }^{3} J=6.9 \mathrm{~Hz}\right), 2.53-2.72(\mathrm{~m}, 4 \mathrm{H})$, $2.45\left(\mathrm{~d}, 1 \mathrm{H},{ }^{3} J=4.8 \mathrm{~Hz}\right), 2.08-2.22(\mathrm{~m}, 2 \mathrm{H}), 1.20-1.25(\mathrm{~m}, 6 \mathrm{H}), 1.20(\mathrm{~s}, 9 \mathrm{H}), 0.88(\mathrm{~s}$, $9 \mathrm{H}), 0.05(\mathrm{~s}, 6 \mathrm{H}) ;{ }^{13} \mathrm{C}$ NMR $\left(100 \mathrm{MHz}, \mathrm{CDCl}_{3}\right): \delta 177.9,72.6,71.3,63.8,47.3,38.9$, $36.7,27.2,25.8,24.0,23.9,18.2,14.3,14.3,-5.5,-5.5$; HRMS (FAB + ) calcd for $\mathrm{C}_{20} \mathrm{H}_{42} \mathrm{O}_{4} \mathrm{~S}_{2} \mathrm{Si}+\mathrm{NH}_{4} 456.2638$ found 456.2660 . 
(1S,2S)-2,2-Dimethylpropionic Acid 1-(2,2-Bis-ethylsulfanylethyl)-3-tert-butyldimethylsilanyloxy)-2-methoxymethoxypropyl Ester.

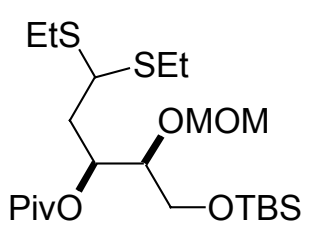

An ice-cooled solution of (1S,2S)-2,2-dimethylpropionic acid 1-(2,2-bis-ethylsulfanylethyl)-3-tert-butyldimethylsilanyloxy)-2-hydroxypropyl ester $(5.10 \mathrm{~g}, 11.6 \mathrm{mmol})$ in dry diisopropylethylamine $(12 \mathrm{~mL})$ was charged with methoxymethyl chloride $(3.5 \mathrm{~mL}, 3.7$ $\mathrm{g}, 46 \mathrm{mmol})$. The mixture was allowed to reach room temperature and was stirred for 16 $\mathrm{h}$, after which time the reaction mixture was quenched with water. Extractive workup and column chromatography (EtOAc/hexanes 1:9) furnished $5.10 \mathrm{~g}$ (94\%) of the product as a colorless oil. $[\alpha]_{\mathrm{D}}-9.0\left(\mathrm{c} 1.3, \mathrm{CHCl}_{3}\right)$; IR (neat) 2958, 1731, 1462, 1281, 1257, 1152, 1031, 920, 838, 777, $669 \mathrm{~cm}^{-1}$; ${ }^{1} \mathrm{H}$ NMR $\left(400 \mathrm{MHz}, \mathrm{CDCl}_{3}\right): \delta 5.34\left(\mathrm{ddd}, 1 \mathrm{H},{ }^{3} J=8.3\right.$ $\left.\mathrm{Hz},{ }^{3} J=4.4 \mathrm{~Hz},{ }^{3} J=4.4 \mathrm{~Hz}\right), 4.80\left(\mathrm{~d}, 1 \mathrm{H},{ }^{2} J=6.7 \mathrm{~Hz}\right), 4.69\left(\mathrm{~d}, 1 \mathrm{H},{ }^{2} J=6.7 \mathrm{~Hz}\right), 3.82$ $\left(\mathrm{dd}, 1 \mathrm{H},{ }^{3} J=8.9 \mathrm{~Hz},{ }^{3} J=6.1 \mathrm{~Hz}\right), 3.74-3.78(\mathrm{~m}, 1 \mathrm{H}), 3.67\left(\mathrm{~d},{ }^{3} J=5.8 \mathrm{~Hz}, 2 \mathrm{H}\right), 3.42(\mathrm{~s}$, $3 \mathrm{H}), 2.56-2,71(\mathrm{~m}, 4 \mathrm{H}), 2.03-2.10(\mathrm{~m}, 1 \mathrm{H}), 2.13-2.19(\mathrm{~m}, 1 \mathrm{H}), 1.21-1.26(\mathrm{~m}, 6 \mathrm{H}), 1.20$ (s, 9H), $0.88(\mathrm{~s}, 9 \mathrm{H}), 0.04(\mathrm{~s}, 3 \mathrm{H}), 0.03(\mathrm{~s}, 3 \mathrm{H}) ;{ }^{13} \mathrm{C}$ NMR $\left(100 \mathrm{MHz}, \mathrm{CDCl}_{3}\right): \delta 177.5$, 97.0, 77.8, 70.9, 62.9, 55.9, 47.6, 38.9, 36.0, 27.2, 25.8, 23.9, 23.6, 18.2, 14.4, 14.3, -5.5, -5.5; HRMS (ES+) calcd for $\mathrm{C}_{22} \mathrm{H}_{46} \mathrm{O}_{5} \mathrm{~S}_{2} \mathrm{Si}+\mathrm{NH}_{4} 500.2900$ found 500.2925.

(1S,2S)-2,2-Dimethylpropionic Acid 1-(2,2-Bis-ethylsulfanylethyl)-3-hydroxy-2methoxymethoxypropyl Ester (17).

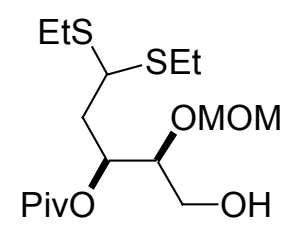

A solution of (1S,2S)-2,2-dimethylpropionic acid 1-(2,2-bis-ethylsulfanylethyl)-3-tertbutyldimethylsilanyloxy)-2-methoxymethoxypropyl ester (5 g, $10.7 \mathrm{mmol})$ was kept in a mixture of THF/water/trifluoracetic acid 17:2:1 $(100 \mathrm{~mL})$ at room temperature overnight. The solution was then neutralized with sat. $\mathrm{K}_{2} \mathrm{CO}_{3}$. Extractive workup with ether and column chromatography (EtOAc/hexanes 3:7) gave $3.6 \mathrm{~g}(94 \%)$ of $\mathbf{1 7}$ as a colorless oil: $[\alpha]_{\mathrm{D}}-40.0\left(\mathrm{c} 1.3, \mathrm{CHCl}_{3}\right)$; IR (neat) 3480, 2967, 2930, 1730, 1480, 1457, 1282, 1153, $1031,919 \mathrm{~cm}^{-1} ;{ }^{1} \mathrm{H}$ NMR $\left(400 \mathrm{MHz}, \mathrm{CDCl}_{3}\right): \delta 5.30-5.34(\mathrm{~m}, 1 \mathrm{H}), 4.70\left(\mathrm{~d}, 1 \mathrm{H},{ }^{2} J=6.8\right.$ $\mathrm{Hz}), 4.64\left(\mathrm{~d}, 1 \mathrm{H},{ }^{2} J=6.8 \mathrm{~Hz}\right), 3.72\left(\mathrm{dd}, 1 \mathrm{H},{ }^{3} J=9.9 \mathrm{~Hz},{ }^{3} \mathrm{~J}=4.7 \mathrm{~Hz}\right), 3.54-3.63(\mathrm{~m}, 2 \mathrm{H})$, 3.44-3.49 (m, 1H), $3.38(\mathrm{~s}, 3 \mathrm{H}), 3.15\left(\mathrm{t}, 1 \mathrm{H},{ }^{3} J=7.1 \mathrm{~Hz}\right), 2.47-2.68(\mathrm{~m}, 4 \mathrm{H}), 2.19$ (ddd, $\left.1 \mathrm{H},{ }^{2} J=14.5 \mathrm{~Hz},{ }^{3} J=9.4 \mathrm{~Hz},{ }^{3} J=4.8 \mathrm{~Hz}\right), 2.00\left(\mathrm{ddd}, 1 \mathrm{H},{ }^{2} J=13.6 \mathrm{~Hz},{ }^{3} J=10.0 \mathrm{~Hz},{ }^{3} J\right.$ $=3.2 \mathrm{~Hz}), 1.17-1.22(\mathrm{~m}, 6 \mathrm{H}), 1.17(\mathrm{~s}, 9 \mathrm{H}) ;{ }^{13} \mathrm{C} \mathrm{NMR}\left(100 \mathrm{MHz}, \mathrm{CDCl}_{3}\right): \delta 178.2,97.3$, 80.8, 70.6, 61.7, 56.0, 47.3, 38.8, 36.3, 27.0, 24.1, 23.6, 14.3, 14.3; HRMS (ES+) calcd for $\mathrm{C}_{16} \mathrm{H}_{32} \mathrm{O}_{5} \mathrm{~S}_{2}+\mathrm{NH}_{4} 386.2035$ found 386.2054 . 
(1S,2R)-2,2-Dimethylpropionic Acid 1-(2,2-Bis-ethylsulfanylethyl)-2methoxymethoxy-3-oxopropyl Ester.

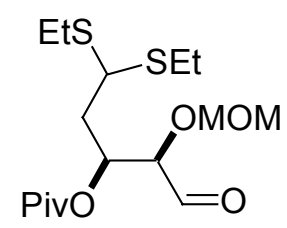

To a solution of alcohol $17(4.00 \mathrm{~g}, 10.9 \mathrm{mmol})$ and pyridine $(3 \mathrm{~mL})$ in methylene chloride $(100 \mathrm{~mL})$ was added freshly prepared Dess-Martin-periodinane $(9.00 \mathrm{~g}, 21$ $\mathrm{mmol}$ ) in one portion and the resulting solution was stirred at room temperature for 10 min. The reaction was immediately monitored by TLC. When the starting material was consumed $(10 \mathrm{~min})$ the mixture was treated with a 1:1 mixture of sat. $\mathrm{NaHCO}_{3} / \mathrm{sat}$. $\mathrm{NaS}_{2} \mathrm{O}_{3}(100 \mathrm{~mL})$ and then neutralized with sat. $\mathrm{NaHCO}_{3}$. After further 10 min of stirring the mixture was extracted with ether. The combined organic layers were dried over $\mathrm{MgSO}_{4}$, filtered and concentrated under reduced pressure. The crude and very unstable aldehyde was directly used in the next step without any purification.

(1S,2S)-2,2-Dimethylpropionic Acid 1-(2,2-Bis-ethylsulfanylethyl)-4,4-dibromo-2methoxymethoxybut-3-enyl Ester.

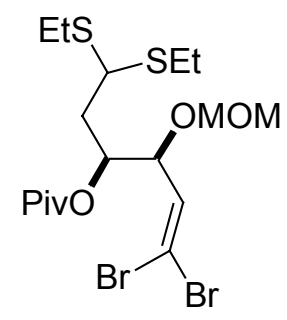

To an ice-cooled solution of carbon tetrabromide $(10.0 \mathrm{~g}, 30 \mathrm{mmol})$ in dry methylene chloride $(100 \mathrm{~mL})$ was added triphenylphosphine $(16.0 \mathrm{~g}, 61 \mathrm{mmol})$ and the resulting orange solution was stirred in an ice-bath for $30 \mathrm{~min}$. The crude aldehyde prepared in the previous step in dry methylene chloride $(20 \mathrm{~mL})$ was added in one portion at the same temperature. The mixture turned deep red. After stirring at $0{ }^{\circ} \mathrm{C}$ for $10 \mathrm{~min}$ the reaction was quenched with water. Extractive workup with ether and column chromatography (EtOAc/hexanes 5:95) gave $4.3 \mathrm{~g}(75 \%)$ of the desired dibromide as a slightly yellow oil. $[\alpha]_{\mathrm{D}}+24.2\left(\mathrm{c} 0.9, \mathrm{CHCl}_{3}\right)$; IR (neat) 2968, 2929, 1733, 1279, 1150,1101, $1082 \mathrm{~cm}^{-1}$; ${ }^{1} \mathrm{H}$ NMR $\left(400 \mathrm{MHz}, \mathrm{CDCl}_{3}\right): \delta 6.37\left(\mathrm{~d}, 1 \mathrm{H},{ }^{3} \mathrm{~J}=9.0 \mathrm{~Hz}\right), 5.30\left(\mathrm{ddd}, 1 \mathrm{H},{ }^{3} \mathrm{~J}=9.2 \mathrm{~Hz},{ }^{3} J\right.$ $\left.=4.7 \mathrm{~Hz},{ }^{3} J=4.7 \mathrm{~Hz}\right), 4.64\left(\mathrm{~d}, 1 \mathrm{H},{ }^{2} J=6.7 \mathrm{~Hz}\right), 4.57\left(\mathrm{~d}, 1 \mathrm{H},{ }^{2} J=6.7 \mathrm{~Hz}\right), 4.52(\mathrm{dd}, 1 \mathrm{H}$, $\left.{ }^{3} J=9.0 \mathrm{~Hz}, 4.7 \mathrm{~Hz}\right), 3.79\left(\mathrm{dd}, 1 \mathrm{H},{ }^{3} J=8.9 \mathrm{~Hz},{ }^{3} \mathrm{~J}=6.1 \mathrm{~Hz}\right), 3.38(\mathrm{~s}, 3 \mathrm{H}), 2.51-2.70(\mathrm{~m}$, $4 \mathrm{H}), 2.06-2.15(\mathrm{~m}, 2 \mathrm{H}), 1.21-1.28(\mathrm{~m}, 6 \mathrm{H}), 1.21(\mathrm{~s}, 9 \mathrm{H}) ;{ }^{13} \mathrm{C}$ NMR $\left(100 \mathrm{MHz}, \mathrm{CDCl}_{3}\right)$ : $\delta$ 177.4, 134.7, 94.7, 94.1, 75.6, 71.6, 56.0, 47.3, 38.9, 35.8, 27.1, 23.9, 23.7, 14.4, 14.3; HRMS (ES+) calcd for $\mathrm{C}_{17} \mathrm{H}_{30} \mathrm{O}_{4} \mathrm{~S}_{2} \mathrm{Br}_{2}+\mathrm{NH}_{4} 538.0296$ found 538.0303 . 
(3Z)-(1S,2S)-2,2-Dimethylpropionic Acid 1-(2,2-Bis-ethylsulfanylethyl)-4-bromo-2methoxymethoxybut-3-enyl Ester (18).

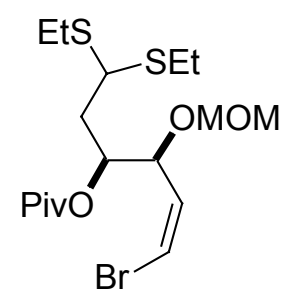

To a solution of (1S,2S)-2,2-dimethylpropionic acid 1-(2,2-bis-ethylsulfanylethyl)-4,4dibromo-2-methoxymethoxybut-3-enyl ester $(5.05 \mathrm{~g}, 9.67 \mathrm{mmol})$ in dry benzene $(100 \mathrm{~mL})$ was added palladium-tetrakis(triphenylphosphine) $(360 \mathrm{mg}, 0.3 \mathrm{mmol})$ and subsequently dropwise charged with tributyltin hydride $(4.4 \mathrm{~mL}, 4.76 \mathrm{~g}, 16 \mathrm{mmol})$. After stirring at room temperature for $2 \mathrm{~h}$ the solvent was removed under reduced pressure and the residue was purified by column chromatography (EtOAc/hexanes 5:95) to give $4.09 \mathrm{~g}$ $(95 \%)$ of the bromide $\mathbf{1 8}$ as a colorless oil. $[\alpha]_{\mathrm{D}}+35.3$ (c $0.8, \mathrm{CHCl}_{3}$ ); IR (neat) 2968, 2929, 1732, 1479, 1280, 1150, 1101, 1030, 920, $734 \mathrm{~cm}^{-1}$; ${ }^{1} \mathrm{H}$ NMR (400 MHz, $\left.\mathrm{CDCl}_{3}\right)$ : $\delta 6.46\left(\mathrm{~d}, 1 \mathrm{H},{ }^{3} J=7.3 \mathrm{~Hz}\right), 6.06\left(\mathrm{dd}, 1 \mathrm{H},{ }^{3} \mathrm{~J}=8.9 \mathrm{~Hz},{ }^{3} J=7.4 \mathrm{~Hz}\right), 5.29-5.34(\mathrm{~m}, 1 \mathrm{H})$, $4.74\left(\mathrm{dd}, 1 \mathrm{H},{ }^{3} J=8.9 \mathrm{~Hz},{ }^{3} J=4.9 \mathrm{~Hz}\right), 4.62\left(\mathrm{~d}, 1 \mathrm{H},{ }^{2} J=6.7 \mathrm{~Hz}\right), 4.56\left(\mathrm{~d}, 1 \mathrm{H},{ }^{2} J=6.7\right.$ $\mathrm{Hz}), 3.78\left(\mathrm{t}, 1 \mathrm{H},{ }^{3} \mathrm{~J}=7.7 \mathrm{~Hz}\right), 3.37(\mathrm{~s}, 3 \mathrm{H}), 2.53-2.69(\mathrm{~m}, 4 \mathrm{H}), 2.09-2.15(\mathrm{~m}, 2 \mathrm{H}), 1.20-$ $1.25(\mathrm{~m}, 6 \mathrm{H}), 1.19(\mathrm{~s}, 9 \mathrm{H}) ;{ }^{13} \mathrm{C} \mathrm{NMR}\left(100 \mathrm{MHz}, \mathrm{CDCl}_{3}\right): \delta 177.5,131.0,112.6,94.7$, 73.5, 71.9, 55.9, 47.3, 38.8, 36.2, 27.2, 24.0, 23.7, 14.4, 14.3. HRMS (ES+) calcd for $\mathrm{C}_{17} \mathrm{H}_{31} \mathrm{O}_{4} \mathrm{~S}_{2} \mathrm{Br}+\mathrm{NH}_{4} 460.1191$ found 460.1204 .

(5Z)-(3S,4S)-6-Bromo-1,1-bis-ethylsulfanyl-4-methoxymethoxyhex-5-en-3-ol.

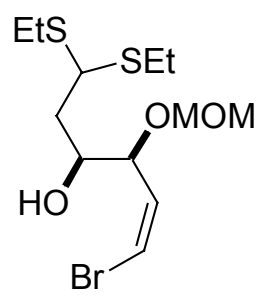

To a solution of 18 (4.09 g, $9.2 \mathrm{mmol})$ in dry methylene chloride $(50 \mathrm{~mL})$ was added dropwise at room temperature a solution of diisopropylaluminum hydride in THF (1M, $28 \mathrm{~mL}, 28 \mathrm{mmol}$ ). The solution was stirred for $1 \mathrm{~h}$ and then quenched with methanol and treated with sat. potassium sodium tartrate solution. Extractive workup with ether and column chromatography (EtOAc/hexanes 2:8) afforded $2.88 \mathrm{~g}(87 \%)$ of the desired alcohol as a colorless oil: $[\alpha]_{\mathrm{D}}+57.8\left(\mathrm{c} 0.8, \mathrm{CHCl}_{3}\right)$; IR (neat) $3461,2963,2926,1622$, $1449,1263,1151,1100,1024,922,726 \mathrm{~cm}^{-1} ;{ }^{1} \mathrm{H}$ NMR $\left(400 \mathrm{MHz}, \mathrm{CDCl}_{3}\right): \delta 6.47(\mathrm{~d}$, $\left.1 \mathrm{H},{ }^{3} J=7.3 \mathrm{~Hz}\right), 6.11\left(\mathrm{t}, 1 \mathrm{H},{ }^{3} J=7.6 \mathrm{~Hz}\right), 4.67\left(\mathrm{~d}, 1 \mathrm{H},{ }^{2} J=6.6 \mathrm{~Hz}\right), 4.58\left(\mathrm{~d}, 1 \mathrm{H},{ }^{2} J=\right.$ $6.7 \mathrm{~Hz}), 4.42\left(\mathrm{dd}, 1 \mathrm{H},{ }^{3} J=8.4 \mathrm{~Hz},{ }^{3} \mathrm{~J}=6.4 \mathrm{~Hz}\right), 4.06\left(\mathrm{dd}, 1 \mathrm{H},{ }^{3} J=10.4 \mathrm{~Hz},{ }^{3} J=4.1 \mathrm{~Hz}\right)$, 3.97-4.00 (m, 1H), $3.38(\mathrm{~s}, 3 \mathrm{H}), 2.78(\mathrm{~s}, \mathrm{br}, 1 \mathrm{H}), 2.49-2.69(\mathrm{~m}, 4 \mathrm{H}), 2.02-2.09(\mathrm{~m}, 1 \mathrm{H})$, 1.78-1.85 (m, 1H), 1.21-1.26 (m, 6H); ${ }^{13} \mathrm{C}$ NMR $\left(100 \mathrm{MHz}, \mathrm{CDCl}_{3}\right): \delta 131.9,112.5$, 94.8, 77.4, 70.7, 55.9, 47.8, 39.1, 24.3, 23.8, 14.5, 14.4. HRMS (ES+) calcd for $\mathrm{C}_{12} \mathrm{H}_{23} \mathrm{O}_{3} \mathrm{~S}_{2} \mathrm{Br}+\mathrm{NH}_{4} 376.0616$ found 376.0607 . 
(5Z)-(3S,4S)-1,1-Bis-ethylsulfanyl-4-methoxymethoxy-8-(trimethylsilanyl)-oct-5-en7-yn-3-ol.

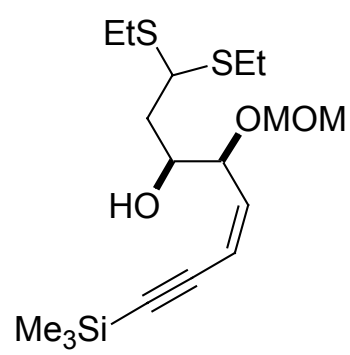

A solution of (3S,4S,5Z)-6-bromo-1,1-bis-ethylsulfanyl-4-methoxymethoxyhex-5-en-3-ol (480 $\mathrm{mg}, 1.34 \mathrm{mmol})$ in degassed diethylamine $(2 \mathrm{~mL})$ was charged with palladiumtetrakis(triphenylphosphine) $(77 \mathrm{mg}, 0.067 \mathrm{mmol})$ and stirred at room temperature for 30 min. Trimethylsilylacetylene $(750 \mu \mathrm{L}, 0.52 \mathrm{mg}, 5.31 \mathrm{mmol})$ was added followed by copper(I)-iodide (25 mg, $0.13 \mathrm{mmol}$ ) and the resulting mixture was stirred overnight. The solvent was removed under reduced pressure and the residue was purified twice by column chromatography (EtOAc/hexanes $2: 8)$ to give $432 \mathrm{mg}(84 \%)$ of the alkyne as a slightly yellow oil. $[\alpha]_{\mathrm{D}}+77.1\left(\mathrm{c} 1.4, \mathrm{CHCl}_{3}\right.$ ); IR (neat) 3473, 2961, 2927, 2149, 1450, 1396, 1250, 1152, 1099, 1027, 845, 760; ${ }^{1} \mathrm{H}$ NMR $\left(400 \mathrm{MHz}, \mathrm{CDCl}_{3}\right): \delta 5.82\left(\mathrm{dd}, 1 \mathrm{H},{ }^{3} J\right.$ $\left.=11.0 \mathrm{~Hz},{ }^{3} J=9.0 \mathrm{~Hz}\right), 5.72\left(\mathrm{~d}, 1 \mathrm{H},{ }^{3} J=11.1 \mathrm{~Hz}\right), 4.67\left(\mathrm{~d}, 1 \mathrm{H},{ }^{2} J=6.6 \mathrm{~Hz}\right), 4.58(\mathrm{~d}$, $\left.1 \mathrm{H},{ }^{2} \mathrm{~J}=6.5 \mathrm{~Hz}\right), 4.43\left(\mathrm{dd}, 1 \mathrm{H},{ }^{3} \mathrm{~J}=8.9 \mathrm{~Hz},{ }^{3} \mathrm{~J}=6.8 \mathrm{~Hz}\right), 4.07\left(\mathrm{dd}, 1 \mathrm{H},{ }^{3} \mathrm{~J}=10.7 \mathrm{~Hz},{ }^{3} \mathrm{~J}=\right.$ $3.9 \mathrm{~Hz}), 3.91-3.97(\mathrm{~m}, 1 \mathrm{H}), 3.36(\mathrm{~s}, 3 \mathrm{H}), 2.83\left(\mathrm{~d}, 1 \mathrm{H},{ }^{3} \mathrm{~J}=2.8 \mathrm{~Hz}\right), 2.52-2.69(\mathrm{~m}, 4 \mathrm{H})$, $1.99-2.03(\mathrm{~m}, 1 \mathrm{H}), 1.80-1.85(\mathrm{~m}, 1 \mathrm{H}), 1.22\left(\mathrm{t}, 3 \mathrm{H},{ }^{3} \mathrm{~J}=7.5 \mathrm{~Hz}\right), 1.22\left(\mathrm{t}, 3 \mathrm{H},{ }^{3} \mathrm{~J}=7.4 \mathrm{~Hz}\right)$, $0.16(\mathrm{~s}, 9 \mathrm{H}) ;{ }^{13} \mathrm{C} \mathrm{NMR}\left(100 \mathrm{MHz}, \mathrm{CDCl}_{3}\right): \delta 140.2,114.2,101.2,100.5,94.9,78.0,70.8$, 55.8, 47.8, 39.1, 24.2, 23.7, 14.4, 14.4, -0.3; HRMS (ES+) calcd for $\mathrm{C}_{17} \mathrm{H}_{32} \mathrm{O}_{3} \mathrm{~S}_{2} \mathrm{Si}\left(\mathrm{M}^{+}\right)$ 376.1562 found 376.1573 .

(5Z)-(3S,4S)-1,1-Bis-ethylsulfanyl-4-methoxymethoxyoct-5-en-7-yn-3-ol (19).

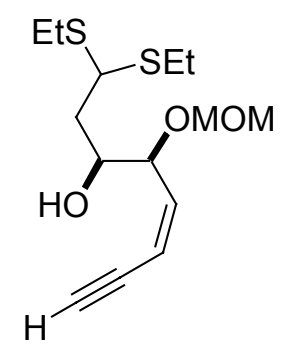

To a solution of (3S,4S,5Z)-1,1-bis-ethylsulfanyl-4-methoxymethoxy-8(trimethylsilanyl)-oct-5-en-7-yn-3-ol (423 $\mathrm{mg}, 1.12 \mathrm{mmol})$ in methanol $(5 \mathrm{~mL})$ was added potassium carbonate $(310 \mathrm{mg}, 2.2 \mathrm{mmol})$ and the resulting slurry was stirred at room temperature for $2 \mathrm{~h}$. Extractive workup of the resulting mixture with ether followed by column chromatography (EtOAc/hexanes 2:8) furnished $330 \mathrm{mg}(96 \%)$ of enyne 19 as a slightly yellow colored oil. $[\alpha]_{\mathrm{D}}+76.8\left(\mathrm{c} 0.8, \mathrm{CHCl}_{3}\right)$; IR (neat) 3471, 3286, 2963, 2927, 1450, 1264, 1151, 1099, 1027, 921, $637 \mathrm{~cm}^{-1} ;{ }^{1} \mathrm{H}$ NMR $\left(400 \mathrm{MHz}, \mathrm{CDCl}_{3}\right): \delta 5.92$ $\left(\mathrm{t}, 1 \mathrm{H},{ }^{3} J=10.1 \mathrm{~Hz}\right), 5.74\left(\mathrm{dd}, 1 \mathrm{H},{ }^{3} J=11.1 \mathrm{~Hz},{ }^{4} J=2.3 \mathrm{~Hz}\right), 4.71\left(\mathrm{~d}, 1 \mathrm{H},{ }^{2} J=6.6 \mathrm{~Hz}\right)$, 
$4.60\left(\mathrm{~d}, 1 \mathrm{H},{ }^{2} J=6.6 \mathrm{~Hz}\right), 4.48\left(\mathrm{dd}, 1 \mathrm{H},{ }^{3} J=9.2 \mathrm{~Hz},{ }^{3} J=6.5 \mathrm{~Hz}\right), 4.09\left(\mathrm{dd}, 1 \mathrm{H},{ }^{3} J=10.5\right.$ $\left.\mathrm{Hz},{ }^{3} J=4.2 \mathrm{~Hz}\right), 3.97-4.01(\mathrm{~m}, 1 \mathrm{H}), 3.39(\mathrm{~s}, 3 \mathrm{H}), 3.16\left(\mathrm{~d}, 1 \mathrm{H},{ }^{4} J=2.3 \mathrm{~Hz}\right), 2.76(\mathrm{~d}, 1 \mathrm{H}$, $\left.{ }^{3} J=4.0 \mathrm{~Hz}\right), 2.55-2.70(\mathrm{~m}, 4 \mathrm{H}), 2.05\left(\mathrm{ddd}, 1 \mathrm{H},{ }^{2} J=14.4 \mathrm{~Hz},{ }^{3} J=10.1 \mathrm{~Hz},{ }^{3} J=4.2 \mathrm{~Hz}\right)$, $1.84\left(\mathrm{ddd}, 1 \mathrm{H},{ }^{2} J=13.5 \mathrm{~Hz},{ }^{3} J=10.7 \mathrm{~Hz},{ }^{3} J=2.1 \mathrm{~Hz}\right), 1.25\left(\mathrm{t}, 3 \mathrm{H},{ }^{3} J=7.4 \mathrm{~Hz}\right), 1.25(\mathrm{t}$, $\left.{ }^{3} J=7.5 \mathrm{~Hz}, 3 \mathrm{H}\right) ;{ }^{13} \mathrm{C} \mathrm{NMR}\left(100 \mathrm{MHz}, \mathrm{CDCl}_{3}\right): \delta 141.0,113.2,94.9,83.4,79.1,77.8$, 71.0, 56.0, 47.9, 39.1, 24.4, 23.8, 14.5, 14.5. HRMS (ES+) calcd for $\mathrm{C}_{14} \mathrm{H}_{24} \mathrm{O}_{3} \mathrm{~S}_{2}+\mathrm{NH}_{4}$ 322.1511 found 322.1521 .

(1E)-2-(2-Iodovinyl)-6-methoxybenzoic Acid (20).<smiles>COc1cccc(/C=C/I)c1C(=O)O</smiles>

Ester 13 (100 mg, $0.24 \mathrm{mmol})$ was dissolved in methylene chloride (1 mL). Trifluoracetic acid $(80 \mu \mathrm{L}, 118 \mathrm{mg}, 1 \mathrm{mmol})$ was added and the solution was stirred at room temperature for $1 \mathrm{~h}$ after which time ether was added and the organic phase was three times extracted with sat. $\mathrm{NaHCO}_{3}$. The aqueous layer was acidified and extractive workup with ether gave $55 \mathrm{mg}(77 \%)$ of crude acid $\mathbf{2 0}$ as a gum which solidified within a few days: $\mathrm{mp} 92-95^{\circ} \mathrm{C}$; IR (KBr) 3061, 2929, 2620, 1723, 1471, 1272, 1065, 937, 809, 760, 696, $619 \mathrm{~cm}^{-1} ;{ }^{1} \mathrm{H}$ NMR $\left(400 \mathrm{MHz}, \mathrm{CDCl}_{3}\right): \delta 8.60(\mathrm{~s}, \mathrm{br}, 1 \mathrm{H}), 7.74\left(\mathrm{~d}, 1 \mathrm{H},{ }^{3} \mathrm{~J}=\right.$ $14.6 \mathrm{~Hz}), 7.41\left(\mathrm{t}, 1 \mathrm{H},{ }^{3} J=8.0 \mathrm{~Hz}\right), 7.07\left(\mathrm{~d}, 1 \mathrm{H},{ }^{3} J=7.7 \mathrm{~Hz}\right), 6.96\left(\mathrm{~d}, 1 \mathrm{H},{ }^{3} J=8.2 \mathrm{~Hz}\right)$, $6.90\left(\mathrm{~d}, 1 \mathrm{H},{ }^{3} \mathrm{~J}=14.7 \mathrm{~Hz}\right), 3.94(\mathrm{~s}, 3 \mathrm{H}) ;{ }^{13} \mathrm{C} \mathrm{NMR}\left(100 \mathrm{MHz}, \mathrm{CDCl}_{3}\right): \delta 170.9,156.9$, 124.1, 138.1, 131.7, 122.7, 118.9, 111.0, 80.3, 56.4; HRMS (ES+) calcd for $\mathrm{C}_{10} \mathrm{H}_{9} \mathrm{O}_{3} \mathrm{I}+\mathrm{H}$ 304.9675 found 304.9652 .

(1E,3Z)-(1S,2S)-2-(2-Iodovinyl)-6-methoxybenzoic Acid 1-(2,2-Bisethylsulfanylethyl)-2-methoxymethoxyhex-3-en-5-ynyl Ester (1).

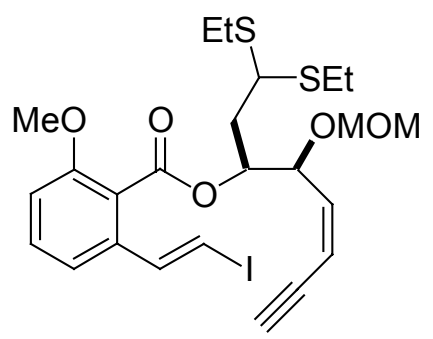

To a solution of acid 20 (300 mg, $987 \mu \mathrm{mol})$ in dry methylene chloride $(4 \mathrm{~mL})$ was at room temperature subsequently added triethylamine $(138 \mu \mathrm{L}, 100 \mathrm{mg}, 988 \mu \mathrm{mol})$ and 2,4,6-trichlorobenzoyl chloride $(156 \mu \mathrm{L}, 244 \mathrm{mg}, 1 \mathrm{mmol})$. The resulting mixture was stirred for $3 \mathrm{~h}$ after which time it was filtered and added to a solution of alkyne 19 $(110 \mathrm{mg}, 361 \mu \mathrm{mol})$ and dimethylaminopyridine $(240 \mathrm{mg}, 2 \mathrm{mmol})$ in dry toluene $(6 \mathrm{~mL})$ at $90{ }^{\circ} \mathrm{C}$. The resulting mixture was stirred at this temperature overnight. Extractive workup with ether followed by column chromatography (EtOAc/hexanes 1:9) afforded $81 \mathrm{mg}(38 \%)$ of ester 1 as a yellow oil: $[\alpha]_{\mathrm{D}}+29.6\left(\mathrm{c} 1.8, \mathrm{CHCl}_{3}\right)$; IR (neat) 3285, 3061, 2964, 2927, 2098, 1732, 1602, 1591, 1571, 1471, 1270, 1151, 1115, 1067, 1028, 947, 
763, $625 \mathrm{~cm}^{-1}$; ${ }^{1} \mathrm{H}$ NMR $\left(400 \mathrm{MHz}, \mathrm{CDCl}_{3}\right): \delta 7.46\left(\mathrm{~d}, 1 \mathrm{H},{ }^{3} \mathrm{~J}=14.9 \mathrm{~Hz}\right), 7.26\left(\mathrm{t}, 1 \mathrm{H},{ }^{3} J\right.$ $=8.1 \mathrm{~Hz}), 6.97\left(\mathrm{~d}, 1 \mathrm{H},{ }^{3} \mathrm{~J}=8.1 \mathrm{~Hz}\right), 6.86\left(\mathrm{~d}, 1 \mathrm{H},{ }^{3} \mathrm{~J}=14.8 \mathrm{~Hz}\right), 6.81\left(\mathrm{~d}, 1 \mathrm{H},{ }^{3} \mathrm{~J}=8.3\right.$ $\mathrm{Hz}), 5.99\left(\mathrm{t}, 1 \mathrm{H},{ }^{3} \mathrm{~J}=10.0 \mathrm{~Hz}\right), 5.82\left(\mathrm{dd}, 1 \mathrm{H},{ }^{3} \mathrm{~J}=11.0 \mathrm{~Hz},{ }^{3} \mathrm{~J}=1.9 \mathrm{~Hz}\right), 5.71(\mathrm{ddd}, 1 \mathrm{H}$, $\left.{ }^{3} J=3.6 \mathrm{~Hz},{ }^{3} J=3.6 \mathrm{~Hz},{ }^{3} J=8.5 \mathrm{~Hz}\right), 4.79\left(\mathrm{dd}, 1 \mathrm{H},{ }^{3} J=5.1 \mathrm{~Hz},{ }^{3} J=8.9 \mathrm{~Hz}\right), 4.69(\mathrm{~d}$, $\left.1 \mathrm{H},{ }^{2} J=6.8 \mathrm{~Hz}\right), 4.60\left(\mathrm{~d}, 1 \mathrm{H},{ }^{2} J=6.8 \mathrm{~Hz}\right), 4.06\left(\mathrm{dd}, 1 \mathrm{H},{ }^{3} \mathrm{~J}=10.3 \mathrm{~Hz},{ }^{3} \mathrm{~J}=4.6 \mathrm{~Hz}\right)$, $3.79(\mathrm{~s}, 3 \mathrm{H}), 3.33(\mathrm{~s}, 3 \mathrm{H}), 3.18\left(\mathrm{~d}, 1 \mathrm{H},{ }^{3} J=1.7 \mathrm{~Hz}\right), 2.58-2.74(\mathrm{~m}, 4 \mathrm{H}), 2.31\left(\mathrm{ddd}, 1 \mathrm{H},{ }^{2} J\right.$ $\left.=14.3 \mathrm{~Hz},{ }^{3} J=8.9 \mathrm{~Hz},{ }^{3} \mathrm{~J}=4.7 \mathrm{~Hz}\right), 2.10\left(\mathrm{ddd}, 1 \mathrm{H},{ }^{3} \mathrm{~J}=14.3 \mathrm{~Hz},{ }^{3} \mathrm{~J}=10.3 \mathrm{~Hz},{ }^{3} \mathrm{~J}=3.6\right.$ $\mathrm{Hz}), 1.22-1.26(\mathrm{~m}, 6 \mathrm{H}) ;{ }^{13} \mathrm{C} \mathrm{NMR}\left(100 \mathrm{MHz}, \mathrm{CDCl}_{3}\right): \delta 166.8,156.1,141.5,140.5$, $136.0,130.5,121.9,117.6,113.6,110.6,94.6,84.0,80.5,78.9,74.2,74.0,55.9,55.8$, 47.5, 37.5, 24.1, 23.7, 14.4, 14.4; HRMS (ES+) calcd for $\mathrm{C}_{24} \mathrm{H}_{31} \mathrm{O}_{5} \mathrm{~S}_{2}+\mathrm{NH}_{4} 608.1001$ found 608.0998 .

(1Z,9Z)-(7S,8S)-7-(2,2-Bis-ethylsulfanylethyl)-4-methoxy-8-methoxymethoxy-8,9dihydro-6-oxabenzocyclododeca-1,9-dien-11-ynone (3).

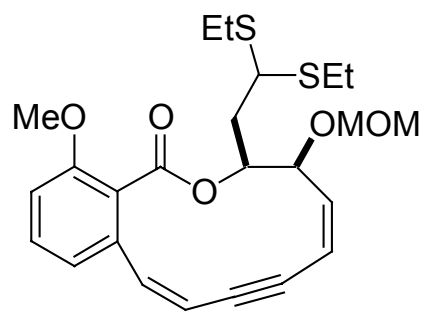

A mixture of ester $1(54 \mathrm{mg}, 91 \mu \mathrm{mol})$, copper(I)-iodide $(2 \mathrm{mg}, 11 \mu \mathrm{mol})$, triphenylphosphine $(6 \mathrm{mg}, 23 \mu \mathrm{mol})$ and $\mathrm{K}_{2} \mathrm{CO}_{3}(22 \mathrm{mg}, 159 \mu \mathrm{mol})$ in dry DMF $(20 \mathrm{~mL})$ was heated at $110^{\circ} \mathrm{C}$ for $26 \mathrm{~h}$. Extractive workup with ether and column chromatography (EtOAc-hexanes 1:9) furnished $13 \mathrm{mg}$ (31\%) of macrocycle 3 as a colorless oil: $[\alpha]_{\mathrm{D}}-156.3$ (c 0.2, $\mathrm{CHCl}_{3}$ ); IR (neat) 2962, 2926, 2852, 1727, 1575, 1471, 1455, 1435, 1277, 1244, 1154, 1034, 972, $794 \mathrm{~cm}^{-1} ;{ }^{1} \mathrm{H}$ NMR (400 MHz, $\left.\mathrm{CDCl}_{3}\right): \delta 7.34\left(\mathrm{t}, 1 \mathrm{H},{ }^{3} \mathrm{~J}=\right.$ $8.0 \mathrm{~Hz}), 6.87\left(\mathrm{~d}, 1 \mathrm{H},{ }^{3} \mathrm{~J}=8.4 \mathrm{~Hz}\right), 6.84\left(\mathrm{~d}, 1 \mathrm{H},{ }^{3} \mathrm{~J}=12.0 \mathrm{~Hz}\right), 6.78\left(\mathrm{~d}, 1 \mathrm{H},{ }^{3} \mathrm{~J}=7.8 \mathrm{~Hz}\right)$, $6.03\left(\mathrm{dd}, 1 \mathrm{H},{ }^{3} \mathrm{~J}=8.4 \mathrm{~Hz},{ }^{3} \mathrm{~J}=10.5 \mathrm{~Hz}\right), 5.86\left(\mathrm{~d}, 1 \mathrm{H},{ }^{3} \mathrm{~J}=11.9 \mathrm{~Hz}\right), 5.67\left(\mathrm{~d}, 1 \mathrm{H},{ }^{3} \mathrm{~J}=\right.$ $10.6 \mathrm{~Hz}), 5.59\left(\mathrm{ddd}, 1 \mathrm{H},{ }^{3} \mathrm{~J}=10.7 \mathrm{~Hz},{ }^{3} \mathrm{~J}=4.4 \mathrm{~Hz},{ }^{3} \mathrm{~J}=1.8 \mathrm{~Hz}\right), 5.29-5.32(\mathrm{~m}, 1 \mathrm{H}), 4.73$ $\left(\mathrm{d}, 1 \mathrm{H},{ }^{2} \mathrm{~J}=6.5 \mathrm{~Hz}\right), 4.68\left(\mathrm{~d}, 1 \mathrm{H},{ }^{2} \mathrm{~J}=6.5 \mathrm{~Hz}\right), 4.32\left(\mathrm{dd}, 1 \mathrm{H},{ }^{3} \mathrm{~J}=11.7 \mathrm{~Hz},{ }^{3} \mathrm{~J}=3.2 \mathrm{~Hz}\right)$, $3.82(\mathrm{~s}, 3 \mathrm{H}), 3.42(\mathrm{~s}, 3 \mathrm{H}), 2.63-2.77(\mathrm{~m}, 4 \mathrm{H}), 2.11-2.24(\mathrm{~m}, 2 \mathrm{H}), 1.25-1.31(\mathrm{~m}, 6 \mathrm{H}) ;{ }^{13} \mathrm{C}$ NMR (100 MHz, $\left.\mathrm{CDCl}_{3}\right): \delta$ 167.0, 158.0, 142.0, 138.7, 137.1, 130.9, 122.8, 121.5, 113.2, 111.1, 111.1, 95.8, 92.1, 91.5, 74.9, 72.8, 56.0, 55.8, 47.8, 34.2, 24.4, 22.7, 14.6, 14.5; HRMS (ES+) calcd for $\mathrm{C}_{24} \mathrm{H}_{30} \mathrm{O}_{5} \mathrm{~S}_{2}+\mathrm{NH}_{4} 480.1878$ found 480.1897 . 

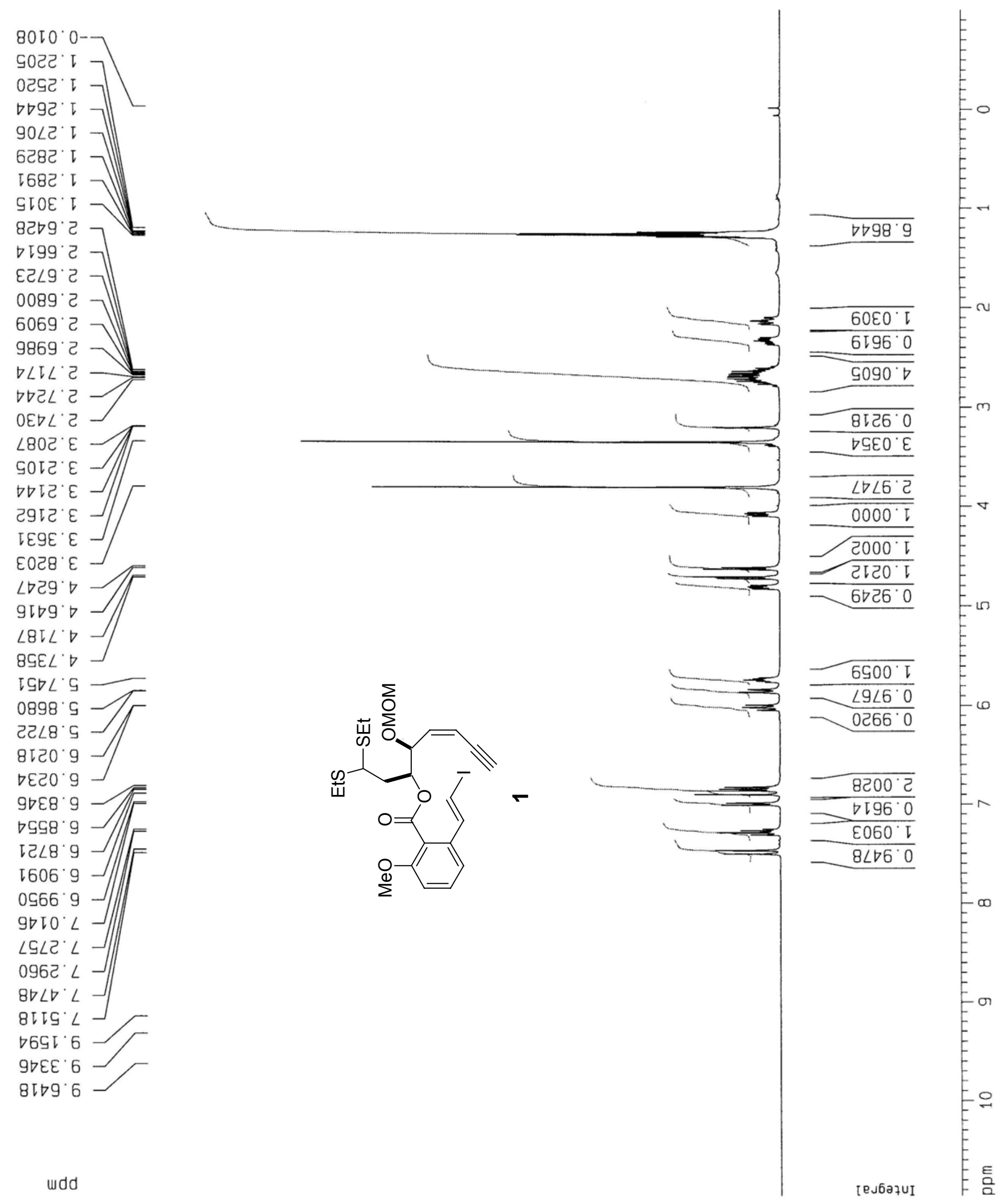


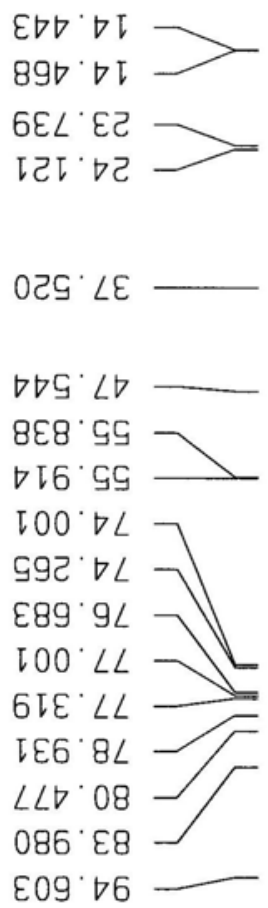

$\varepsilon 09^{\circ} \nabla 6$

$089^{\circ}$ OโT—

$\nabla \nabla 9^{\circ} \varepsilon \downarrow$

Eट9 $\angle I$ -

จ酒纪坃

OLG OEL——

O॰0 $9 \varepsilon \tau$

ESG ODI

$\angle 6 \nabla^{\circ} \tau \nabla$

ह8 ' 99 I

8ะ8 99 น

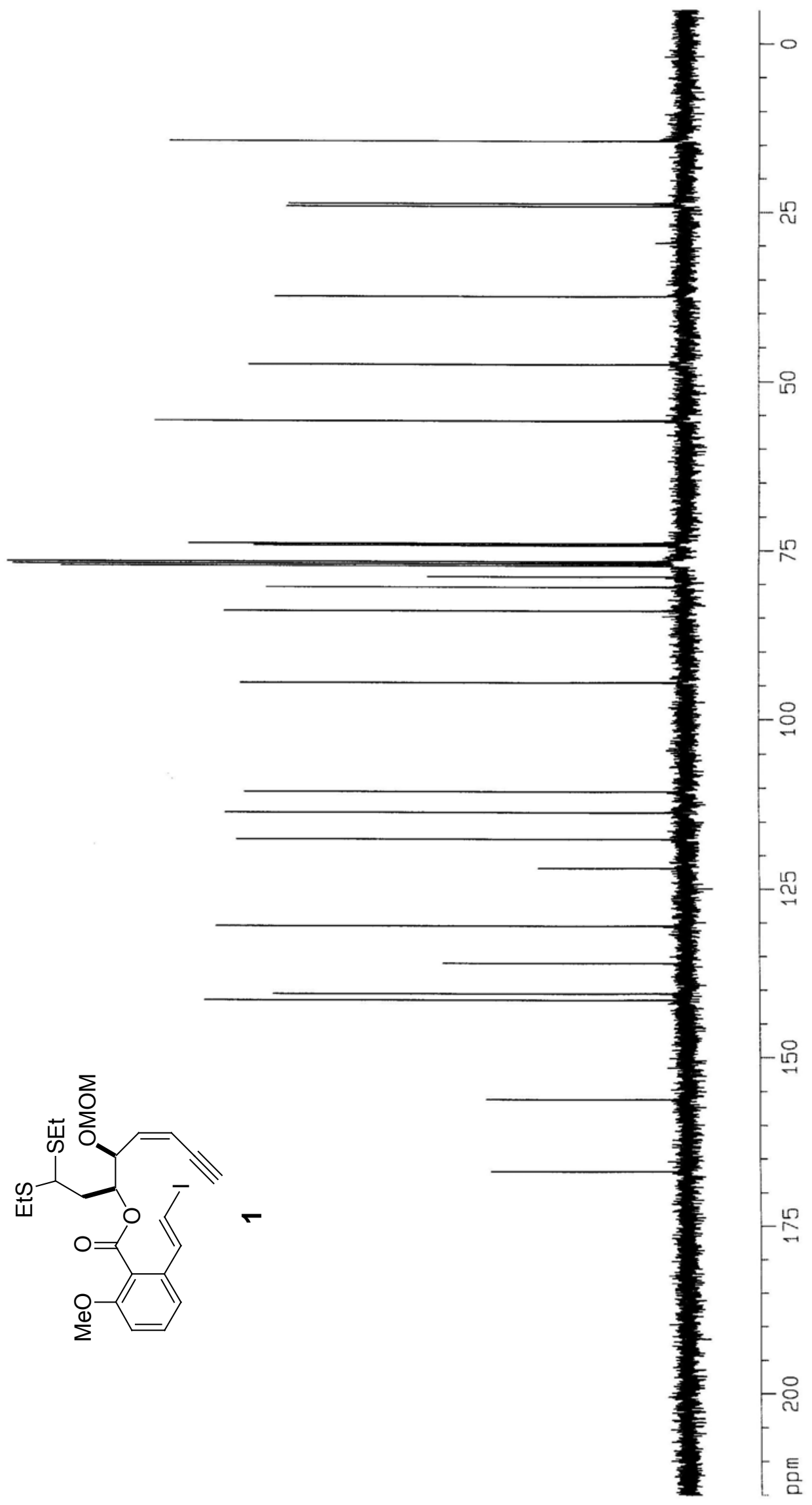



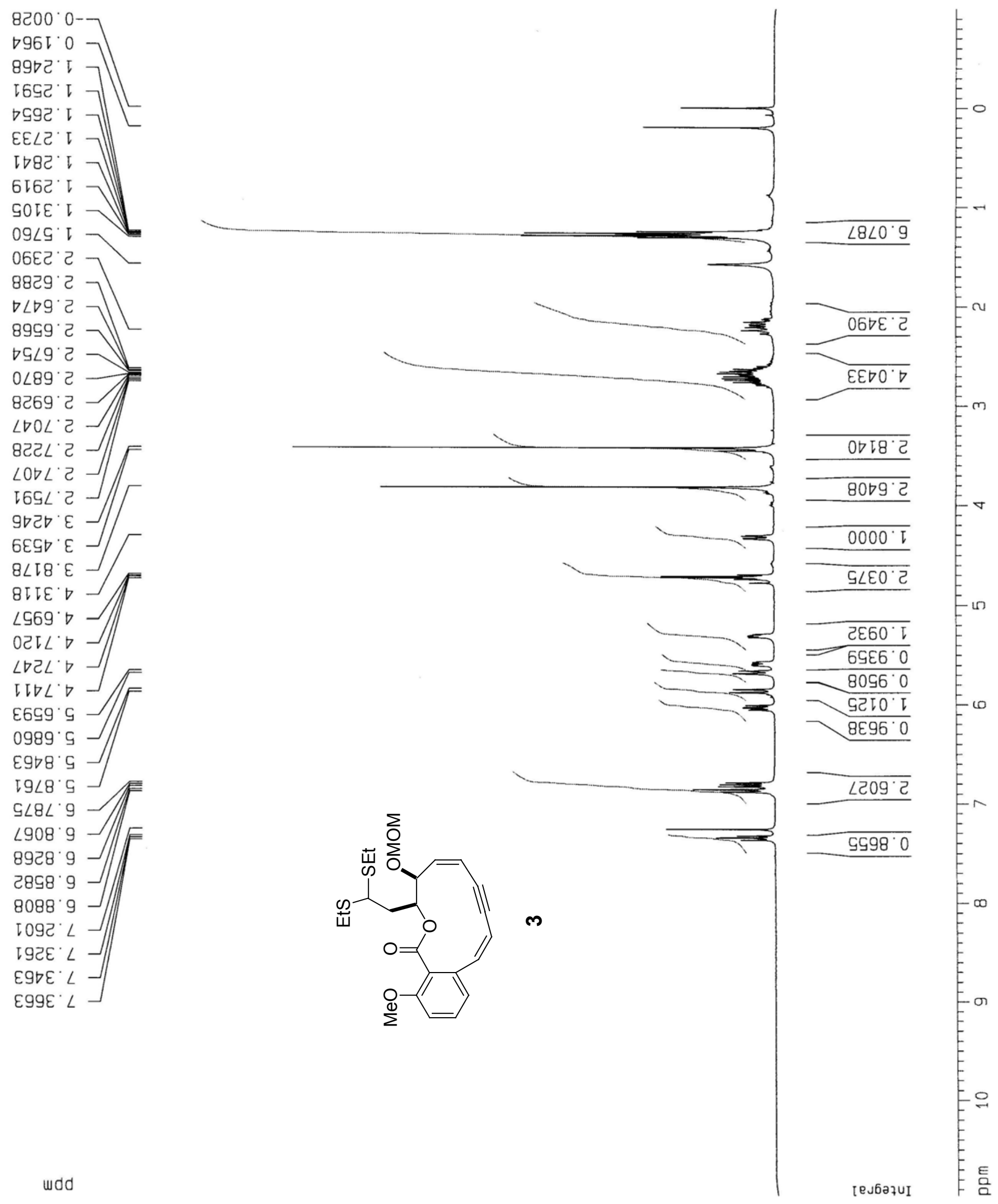

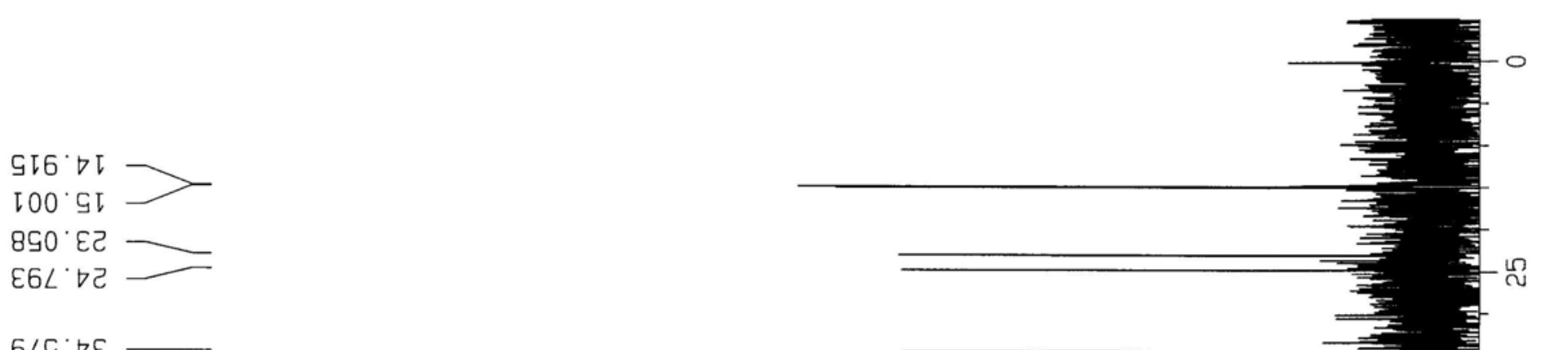

$6 \angle G^{\circ} \nabla E$
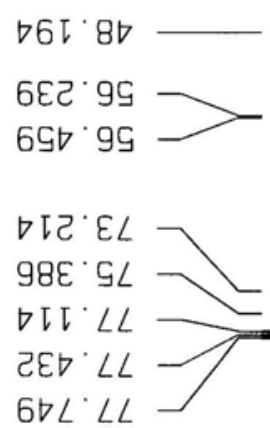

OVLLL

E泀' ᄂ6

$96 b^{\circ} 26$

เ0ट' 96

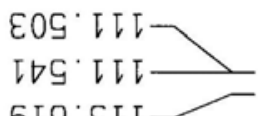

6I9 EIT-

$\angle 0{ }^{\prime} \varepsilon 己 \longleftarrow$

EGE VEโ

OS. $\angle E I-$

$\angle I \cdot 6 E \downarrow-$

$6 \angle \nabla^{\circ} 2 \nabla \downarrow-$

$9 \tau \cdot 891$

$\angle \nabla^{\circ} \angle 9 l$

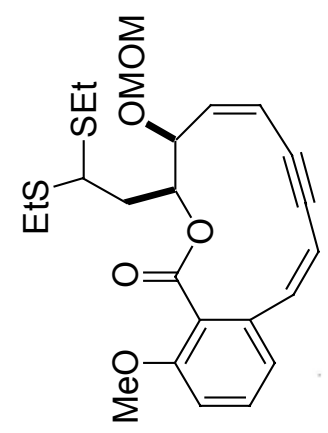

$m$

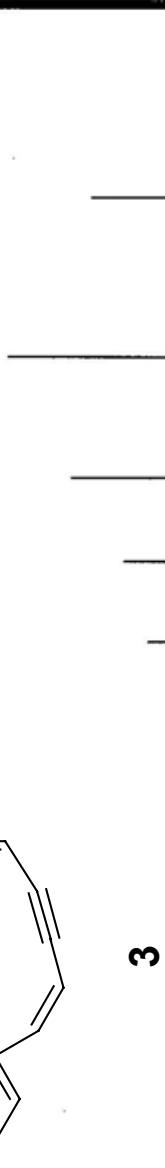




\section{${ }^{3} J(H, H)$ Coupling Constant Calculations}

The predicted vicinal coupling constants $\left({ }^{3} J(\mathrm{H}, \mathrm{H})\right)$ for structures 21, 22, 27 and 28 were obtained by calculating and summing the four individual Ramsey contributions (DSO, PSO, FC and SD; Table S1). In each case the MMFF global minimum geometry (see next section) was employed to obtain the vicinal coupling constant using the B3LYP/6$311 \mathrm{G}(\mathrm{d}, \mathrm{p})$ model as previously described. ${ }^{4}$ Since the predicted ${ }^{3} J(\mathrm{H}, \mathrm{H})$ 's are somewhat higher than the experimental values, we scaled the predictions by employing cis- and trans-acrylonitrile (geometry optimized with the B3LYP/6-311G(d,p) model) as standards. In this way, the values for $\mathbf{2 1}$ and $\mathbf{2 2}$ are reduced to 11.8 and $15.9 \mathrm{~Hz}$, respectively, in complete accord with the experiment (Table S1).

Table S1. Ramsey contributions and ${ }^{3} J(\mathrm{H}, \mathrm{H})$ total for $\mathrm{C} 8-\mathrm{C} 9$ with the DFT B3LYP/6-311G(d,p) model; Hz.

\begin{tabular}{llllllll}
\hline & DSO & PSO & FC & SD & Total & Exp $^{\mathrm{a}}$ & $\Delta^{3} J(\mathrm{H}, \mathrm{H})$ \\
\hline $\mathbf{2 1}$ & -0.002 & -0.57 & 14.78 & -0.052 & 14.2 & “11.8” & \\
$\mathbf{2 2}$ & -2.74 & 3.02 & 19.72 & 0.39 & 20.4 & “15.9” & \\
cis- $\mathbf{C H}_{2}=\mathbf{C H C N}$ & -0.76 & 0.072 & 14.89 & -0.043 & 14.2 & 11.8 & -2.4 \\
trans $\mathbf{C H}=\mathbf{C H C N}$ & -3.30 & 4.77 & 20.56 & 0.33 & 22.4 & 17.9 & -4.5 \\
$\mathbf{2 7}$ & -2.84 & 3.05 & 19.64 & 0.40 & 20.2 & “15.7” & \\
$\mathbf{2 8}$ & -2.81 & 4.39 & 19.67 & 0.40 & 21.7 & "17.2” & \\
\hline
\end{tabular}

${ }^{a}$ i) http://chemistry.utah.edu/faculty/vogler/LectureNotes331/CH331Chapter13.pdf; ii) Lambert, J. B.; Shurvell, H. F.; Lightner, D. A.; Cooks, R. G. In Organic Structural Spectroscopy; Prentice Hall, Englewood Cliffs, N.J., 1998, p 72.

To test whether the methoxy group in the aromatic ring might be responsible for the reduction of ${ }^{3} J(\mathrm{H}, \mathrm{H})$ from 16 to $12 \mathrm{~Hz}$ in trans-22, the vicinal coupling constants for the truncated structures $\mathbf{2 7}$ (model for $\mathbf{2 2}$ ) and $\mathbf{2 8}$ (model for $\mathbf{3}$ ) at the same geometries as the full structures were were calculated to be 20.2 and $21.7 \mathrm{~Hz}$, respectively. Scaled by the value for trans-acrylonitrile, the latter fall to 15.7 and $17.2 \mathrm{~Hz}$, respectively (Table S1). The experimental value of $16 \mathrm{~Hz}$ for trans-oxamidines is not only reproduced by the $J$ calculations; it is also unperturbed by the aromatic OMe substitution. Thus, we conclude that the accurately reproduced value of $12 \mathrm{~Hz}$ for the cis-structure, along with the 
thermodynamic relationships captured by text Table 2 and Table $\mathbf{S 2}$ below, is fully supportive of $E$ to $Z$ rearrangement at C8-C9 during formation of $\mathbf{3}$.

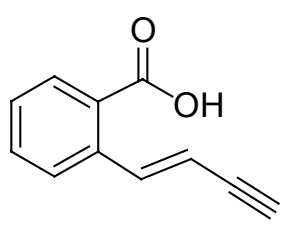

27<smiles>C#C/C=C/c1cccc(OC)c1C(=O)O</smiles>

28

\section{Energy Calculations}

Structures 21-26 cited in Tables $\mathbf{2}$ and $\mathbf{S 2}$ were obtained by Monte Carlo conformational searching with the MMFF force field implemented in MacroModel6.5. ${ }^{5}$ Each structure was searched for 3000 steps with an $6.0 \mathrm{kcal} / \mathrm{mol}$ energy cap resulting in the number of conformations and number of times the global minima (glomin) were located cited in Table S2. That the conformational searches are complete is indicated by the large number of times the glomin were found (Table S2). Generally, 5-15 is sufficient to assure completeness. ${ }^{6}$ In each case the lowest energy structure was further subjected to single point energy calculations using the DFT and MP2 methods, B3LYP/6311G(d,p)//MMFF and MP2/6-311G(d,p)//MMFF, respectively, in Gaussian 03. ${ }^{7}$ The absolute and relative energies are recorded in Table $\mathbf{S 2}$.

Table S2. Single point DFT and MP2 energies of annelated and unsaturated 12-membered ring lactones optimized with the MMFF force field.

\begin{tabular}{ccccccc}
\cline { 2 - 7 } & $\begin{array}{c}\text { No. } \\
\text { Confs }\end{array}$ & $\begin{array}{c}\text { Glomin } \\
\text { Found }^{\mathrm{a}}\end{array}$ & $\begin{array}{c}\mathrm{DFT} \\
\mathrm{au}^{\mathrm{b}}\end{array}$ & $\begin{array}{c}\Delta \mathrm{E}(\mathrm{rel}), \\
\mathrm{kcal}^{\mathrm{c}}\end{array}$ & $\begin{array}{c}\mathrm{MP} 2, \\
\mathrm{Au}^{\mathrm{d}}\end{array}$ & $\begin{array}{c}\Delta \mathrm{E}(\mathrm{rel}), \\
\mathrm{Kcal}^{\mathrm{c}}\end{array}$ \\
\hline $\mathbf{2 1}$ & 2 & 484 & -729.365592 & 0.0 & -727.254772 & 0.0 \\
$\mathbf{2 2}$ & 4 & 351 & -729.342626 & 14.4 & -727.229779 & 15.7 \\
$\mathbf{2 3}$ & 10 & 273 & -730.589678 & 0.0 & -728.465345 & 0.0 \\
$\mathbf{2 4}$ & 4 & 323 & -730.577734 & 7.5 & -728.450978 & 9.0 \\
$\mathbf{2 5}$ & 5 & 390 & -730.604175 & 2.6 & -728.473487 & 2.4 \\
$\mathbf{2 6}$ & 4 & 308 & -730.608372 & 0.0 & -728.477306 & 0.0 \\
\hline
\end{tabular}

${ }^{a}$ Number of times global minimum located; ${ }^{b} \mathrm{~B} 3 \mathrm{LYP} / 6-311 \mathrm{G}(\mathrm{d}, \mathrm{p}) / / \mathrm{MMFF} ;{ }^{\mathrm{c}}$ Only global minima compared; ${ }^{\mathrm{M}} \mathrm{MP} 2 / 6-311 \mathrm{G}(\mathrm{d}, \mathrm{p}) / / \mathrm{MMFF}$. 
(1) Fresko, J. N.; Morrow, G. W.; Swenton, J. S. J. Org. Chem. 1985, 50, 805-810.

(2) Kim, J. Y.; Nam Shin, J. E.; Chun, K. H. Bull. Korean Chem. Soc. 1996, 17, 478-480.

(3) Wong, M. Y. H.; Gray, G. R. J. Am. Chem. Soc. 1978, 100, 3548-3553.

(4) For definitions of the various terms and the method of calculation, see: a) Peralta, J. E.; Barone, V.; Contreras, R. H.; Zaccari, D. G.; Snyder, J. P. J. Am. Chem. Soc. 2001, 123, 9162-9163; b) Barone V.; Peralta, J. E.; Contreras, R. H.; Snyder, J. P. J. Phys. Chem. A 2002, 106, 5607-5612.

(5) Halgren, T. A.; Nachbar, R. B. J. Comput. Chem. 1996, 17, 587-615; b) Halgren, T. A. J. Comput. Chem. 1999, 20, 730-748; c) cf. www.schrodinger.com/Products/macromodel.html

(6) a) Chang, G.; Guida, W. C.; Still, W. C. J. Am. Chem. Soc. 1989, 111, 4379-4386; b) Goodman, J. M.; Still, W. C J. Comput. Chem. 1991, 12, 1110-1117; footnote 5.

(7) Gaussian 03, Revision A.1, M. J. Frisch, G. W. Trucks, H. B. Schlegel, G. E. Scuseria, M. A. Robb, J. R. Cheeseman, J. A. Montgomery, Jr., T. Vreven, K. N. Kudin, J. C. Burant, J. M. Millam, S. S. Iyengar, J. Tomasi, V. Barone, B. Mennucci, M. Cossi, G. Scalmani, N. Rega, G. A. Petersson, H. Nakatsuji, M. Hada, M. Ehara, K. Toyota, R. Fukuda, J. Hasegawa, M. Ishida, T. Nakajima, Y. Honda, O. Kitao, H. Nakai, M. Klene, X. Li, J. E. Knox, H. P. Hratchian, J. B. Cross, C. Adamo, J. Jaramillo, R. Gomperts, R. E. Stratmann, O. Yazyev, A. J. Austin, R. Cammi, C. Pomelli, J. W. Ochterski, P. Y. Ayala, K. Morokuma, G. A. Voth, P. Salvador, J. J. Dannenberg, V. G. Zakrzewski, S. Dapprich, A. D. Daniels, M. C. Strain, O.

Farkas, D. K. Malick, A. D. Rabuck, K. Raghavachari, J. B. Foresman, J. V. Ortiz, Q. Cui, A. G. Baboul, S. Clifford, J. Cioslowski, B. B. Stefanov, G. Liu, A. Liashenko, P. Piskorz, I. Komaromi, R. L. Martin, D. J.

Fox, T. Keith, M. A. Al-Laham, C. Y. Peng, A. Nanayakkara, M. Challacombe, P. M. W. Gill, B. Johnson, W. Chen, M. W. Wong, C. Gonzalez, and J. A. Pople, Gaussian, Inc., Pittsburgh PA, 2003. 